EPJ Web of Conferences 59, 16005 (2013)

DOI: $10.1051 /$ epjconf/20135916005

(C) Owned by the authors, published by EDP Sciences, 2013

\title{
Evaluation of electrical conductivity for copper foam/plasma using isochoric pulsed-power discharge
}

\author{
Yusuke Amano, Yasutoshi Miki, Takuya Takahashi, Toru Sasaki, Takashi \\ Kikuchi and Nob. Harada
}

\author{
Nagaoka University of Technology, Kamitomioka 1603-1, Nagaoka 940-2188, Japan
}

\begin{abstract}
Electrical conductivity in warm dense state has been evaluated by using isochoric pulsed-power discharge. The isochoric heating can be achieved by surrounding the foamed metal with a sapphire capillary as a rigid wall. The electrical conductivity and the temperature of copper foam/plasma have been evaluated by the applied voltage-current waveform and emission intensity. Observed electrical conductivity and foam/plasma temperature is about $10^{4} \mathrm{~S} / \mathrm{m}$ and $4000 \mathrm{~K}$ in $0.10 \rho_{s}$.
\end{abstract}

\section{INTRODUCTION}

Warm dense matter (WDM) is of key interest to understand the formation of giant planets and/or hydrodynamics of fuel pellet in inertial confinement fusion. WDM region is defined that the density goes from $10^{-3} \rho_{\mathrm{s}}\left(\rho_{\mathrm{s}}\right.$ is the solid density of matter) to $10 \rho_{s}$, and temperature varies from 0.1 to $10 \mathrm{eV}$. The characteristics of WDM are hard to study theoretically from first principle approach. To create and to characterize properly the WDM condition are difficult in a laboratory. For this reason, electrical conductivity in the WDM region is unclear [1-15].

In this study, we propose an evaluation of electrical conductivity in WDM state by using pulsedpower discharge with isochoric heating. The features of the method are the possibility to produce isochoric conditions, the use of conventional tamper, avoidance of skin effect, and direct spectroscopic measurement [16]. Thus, the temperature dependence of electrical conductivity in WDM state can be easily evaluated by the pulsed-power discharge with isochoric heating. The temperature dependence of electrical conductivity in WDM is neither metallic nor ideal plasma.

\section{EXPERIMENTAL SETUP}

Figure 1 (a) shows a schematic diagram of isochoric pulsed-power discharge system. To achieve isochoric heating, copper foam with from $50 \mu \mathrm{m}$ to $600 \mu \mathrm{m}$ of porous sizes and about $90 \%$ porosity, was packed into a sapphire capillary $(\phi 5 \times 10 \mathrm{~mm})$. To avoid the surface creepage on sapphire, turbomolecular and rotary pumps are set at bottom of chamber. The interior of the chamber pressure is set to be less than $10^{-3} \mathrm{~Pa}$. The density of WDM can be controlled by enclosed volume of copper foam.

Figure 1 (b) shows an equivalent circuit of experimental setup. The capacitors $(3 \times 1.87 \mu \mathrm{F})$ were charged up to $15 \mathrm{kV}$ to ensure the vaporization of copper foam. Time evolutions of current $I(\mathrm{t})$ and voltage $V(\mathrm{t})$ in foam/plasma were measured by a Rogowski coil and high voltage probes (Tektronix: P6015A). The obtained voltage was composed of resistive and inductive parts. The stray inductance $L$ of the discharge device was estimated to be $165 \mathrm{nH}$ from the preliminary experiment with the

This is an Open Access article distributed under the terms of the Creative Commons Attribution License 2.0, which permits unrestricted use, distribution, and reproduction in any medium, provided the original work is properly cited. 


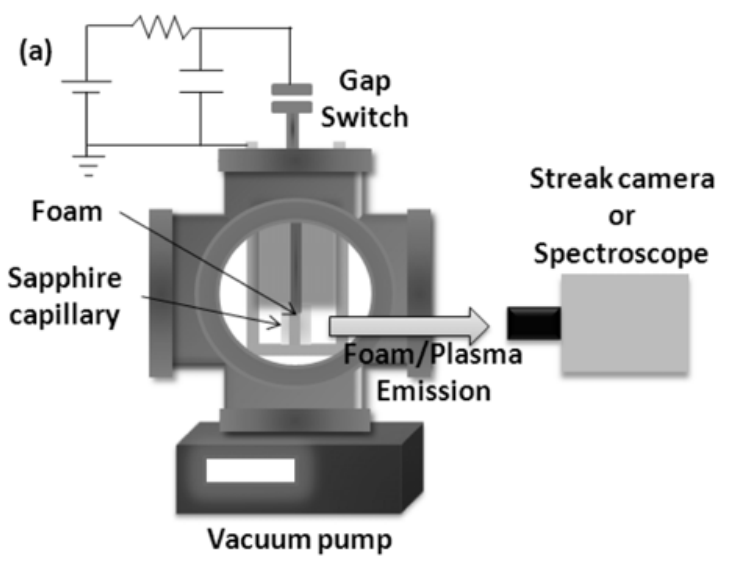

(b)

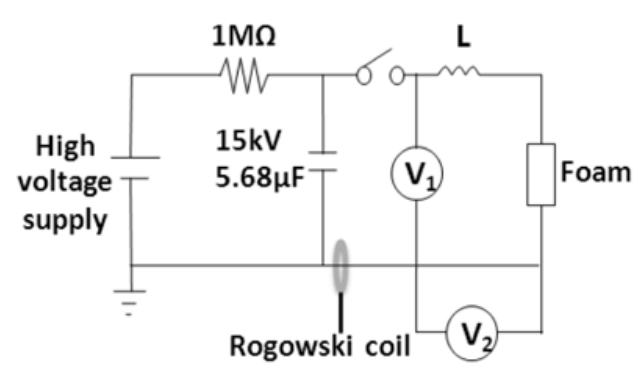

Figure 1. Experimental setup. (a) Schematic diagram of pulsed-power discharge with isochoric heating. (b) Equivalent circuit.

short-circuit. The applied voltage of foam/plasma $V_{\text {foam }}(\mathrm{t})$ is estimated to be

$$
V_{\text {foam }}(t)=\left(V_{1}(t)-V_{2}(t)\right)-L \frac{d I(t)}{d t},
$$

where, $V_{1}(\mathrm{t})$ is the voltage between gap switch and ground, $V_{2}(\mathrm{t})$ is the voltage between the return cable and ground. The input energy $E(t)$ in foam/plasma is also estimated to be

$$
E(t)=\int_{0}^{t} V_{\text {foam }}(t) I(t) d t
$$

The electrical conductivity $\sigma(t)$ in foam/plasma is evaluated by the following equation;

$$
\sigma(t)=\frac{l}{\pi r^{2}} \frac{I(t)}{V_{\text {foam }}(t)},
$$

where $l$ is length of foam/plasma and $r$ is the foam/plasma radius.

To evaluate the temperature without equation of state models, the foam/plasma emission is measured by the spectroscope (Hamamatsu Photonics: C1119-01) mounted on a streak camera (Hamamatsu Photonics: C7700-1). The foam/plasma temperature is estimated by using line pair method;

$$
\ln \left(\frac{\varepsilon_{21} \lambda_{21}}{A_{21} g_{2}}\right)=-\frac{E_{2}}{k_{B} T}+\ln K,
$$

where $\lambda_{21}$ is the wavelength, $A_{21}$ is the spontaneous emission coefficient, $\varepsilon_{21}$ is the emission factor of the spectral line, $E_{2}$ is the upper level energy, $T$ is the foam/plasma temperature, $k_{B}$ is the Boltzmann constant, and $K$ is the constant factor.

\section{EXPERIMENTAL RESULTS}

Figure 2 shows typical waveforms of (a) the voltage and current, (b) emission spectrum at $9 \mu \mathrm{s}$ from beginning discharge, (c) the input energy and foam/plasma temperature, and (d) the electrical conductivity in $0.10 \rho_{\mathrm{s}}$. The peak voltage and current as shown in Fig. 2 (a) are $1 \mathrm{kV}$ and $50 \mathrm{kA}$, respectively. Figure 2 (b) shows that the foam/plasma emission seems from not only excited atoms but also ionized atoms at $651 \mathrm{~nm}$ [17]. Figure 2 (c) shows typical evolution of input energy estimated by voltage and current waveforms. In case of $0.10 \rho_{\mathrm{s}}$, the evaporation energy is estimated to be $300 \mathrm{~J}$. From input energy history, the copper foam is sure to be vaporized at $8 \mu$ s from beginning of discharges. 

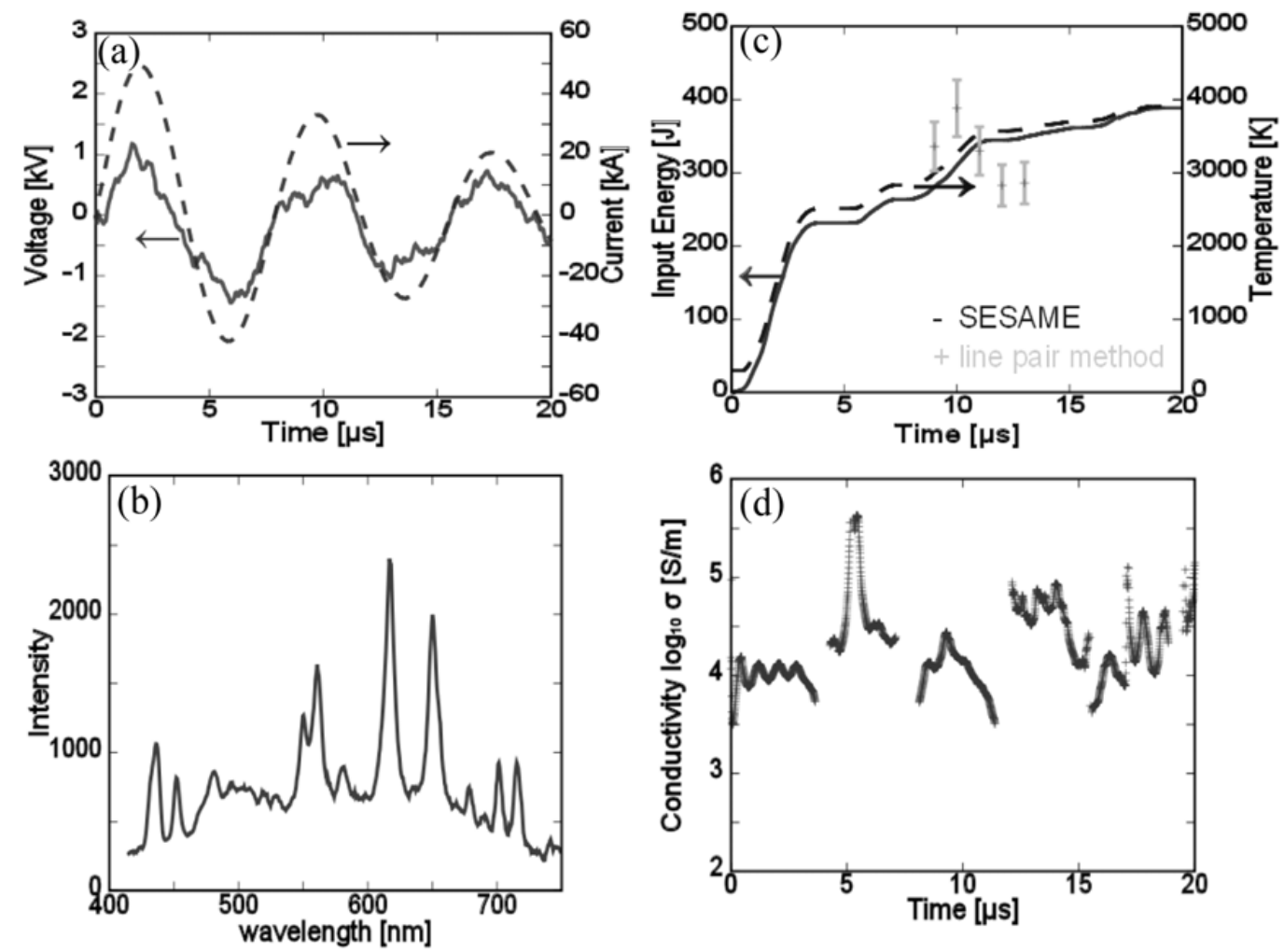

Figure 2. Typical waveforms of (a) voltage and current, (b) emission spectrum at $9 \mu$ s from the beginning of discharge (c) input energy and typical temperature estimated by line pear method and SESAME equation of state table Cu3333, and (d) electrical conductivity.

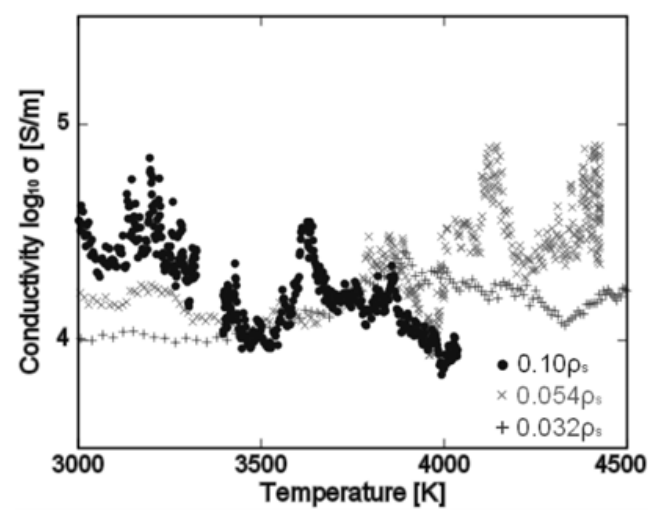

Figure 3. Foam/plasma temperature vs. electrical conductivity in $0.10 \rho_{\mathrm{s}}, 0.054 \rho_{\mathrm{s}}$, and $0.032 \rho_{\mathrm{s}}$.

Figure 2 (c) also shows the evolution of temperature estimated by the SESAME [18] with input energy history and by the line pair method using spectra of $\mathrm{Cu}$ I at $477 \mathrm{~nm}$ and $617 \mathrm{~nm}$ with eq. (4) [19]. The observed spectrum as shown in Fig. 2 (b) has not only the narrow spectrum from emission of copper but also broader spectrum. However, the broad spectrum indicates the optically thick, and the optical depth is not enough to assume the black body, that is, the obtained spectrum depends on the wavelength. 
To evaluate this emission, we should know the emissivity and the opacity of medium. Thus, the evaluation of the WDM temperature is made by the line pair method. From this comparison, the experimentally observed and the numerically evaluated temperatures are almost the same between $8 \mu \mathrm{s}$ and $13 \mu \mathrm{s}$.

As a result, it is confirmed that the copper foam became the WDM state by the pulsedpower discharge device with isochoric heating. The electrical conductivity of copper foam/plasma is estimated to be about $10^{4} \mathrm{~S} / \mathrm{m}$. The observed electrical conductivity is in agreement with the other experiments [2,9] and predictions [5].

Figure 3 shows the electrical conductivity of copper as a function of temperature in case of $0.10 \rho_{\mathrm{s}}$, $0.054 \rho_{\mathrm{s}}$, and $0.032 \rho_{\mathrm{s}}$. Generally, in the case of metal, the temperature dependence of the electrical conductivity is proportional to $\mathrm{T}^{-1}$. In the case of ideal plasma, it is proportional to $\mathrm{T}^{3 / 2}$. As shown in Fig. 3, the dependence of the electrical conductivity here is neither metallic nor ideal plasma.

\section{CONCLUSION}

We have evaluated electrical conductivity in WDM state by using pulsed-power discharge with isochoric heating. To evaluate the foam/plasma temperature without equation of state models, the foam/plasma emission was measured by the spectroscope. The result indicated that the foam/plasma temperature obtained by the line pair method is comparable with the temperature estimated by the input energy history with SESAME. Observed electrical conductivity and foam/plasma temperature is about $10^{4} \mathrm{~S} / \mathrm{m}$ and $4000 \mathrm{~K}$ in $0.1 \rho_{\mathrm{s}}$. The observed electrical conductivity is in agreement with the other experiments and predictions. The temperature dependence of electrical conductivity is neither metallic nor ideal plasma.

This work was partly supported by Grant-in-Aid for Young Scientists (B) from Japan Society for the Promotion of Science (23740406).

\section{References}

[1] R. P. Drake, High-Energy-Density Physics, Springer (2006)

[2] A.W. DeSilva and J. D. Katsouros, Phys. Rev. E 57, 5945 (1998)

[3] A.W. DeSilva and H.-J. Kunze, Phys. Rev. E 58, 6557 (1998)

[4] P. Renaudin, C Blancard, G. Faussurier, and P. Noiret, Phys. Rev. Lett. 88, 215001 (2002)

[5] M.P. Desjarlais, Contrib. Plasma Phys. 41, 267 (2001)

[6] M. P. Desjarlais, J. D. Kress, and L. A. Colins, Phys. Rev. E 66, 025401(2002)

[7] T. Sasaki, Y. Yano, M. Nakajima, T. Kawamura, and K. Horioka, Laser Part. Beams 24, 371 (2006)

[8] T. Sasaki, Y. Yano, M. Nakajima, T. Kawamura, and K. Horioka, Journal of Physics: Conference Series 112, 042027 (2008)

[9] T. Sasaki, M. Nakajima, T. Kawamura, and K. Horikoka, Phys. Plasmas, 17, 084501(2010)

[10] P. A. Ni, F. M. Bieniosek, M. Leitner, C. Weber, and W. L. Waldron, Nucl. Instrum. Methods Phys. Res. A 606, 169-171 (2009)

[11] J.J. Barnard, et. al., Nucl. Instrum. Methods Phys. Res. A 577, 275 (2007)

[12] S. Kuhlbrodt, and R. Redmer, Phys. Rev. E 62, 7191 (2000)

[13] R. Redmer, Phys. Rev. E 59, 1073 (1999)

[14] H. Yoneda, H. Morikami, K. Ueda, and R. M. More, Phys. Rev. Lett. 91, 075004 (2003) 


\section{IFSA 2011}

[15] A. Mancic, et. al., High Energy Density Physics 6, 21 (2010)

[16] Y. Amano, et. al., Rev. Sci. Instrum. 83, 085107 (2012)

[17] M. Fu, M. Jogwich, M. Knebel, and K. Wiesemann, Atomic Data and Nuclear Data Tables 61, 1 (1995)

[18] S. P. Lyon, J. D. Johnson, T-1 Handbook of the SESAME Equation of State Library, LA-CP-98100 (1998)

[19] NIST Atomic Spectra Database Lines Form 